\title{
Indicadores sociodemográficos y seguridad alimentaria en adultos mayores. Zacatecas, México
}

\author{
Flor de María Sánchez Morales, Rafael Samaniego Garay \\ e Isauro García Alonzo \\ Universidad Autónoma de Zacatecas (México)
}

\begin{abstract}
Objetivo. Identificar y comparar asociación entre indicadores sociodemográficos y seguridad alimentaria (SA) en dos grupos de adultos mayores: trabajadores del estado y trabajadores informales o autoempleados. Método: Investigación transversal analítica y comparativa en $>60$ años, muestreo simple aleatorio y estratificado por edad (60-70, 71-80 y >81) integrada por 134 y 123 sujetos para primero y segundo grupo. Se aplicó cuestionario ad hoc para datos sociodemográficos. Regla AMAI, para Nivel Socioeconómico y Escala Latinoamericana y Caribeña para seguridad alimentaria (ELCSA). Resultados: predominio femenino en ambas instituciones (56.7\% y 74.0\%; edad promedio 68.5 y 67.8 años, respectivamente). En la Inseguridad Alimentaria hubo diferencia significativa entre ambos grupos $(p=0.001)$, en los burócratas predominó inseguridad leve con $91 \%$ y moderada para los informales, $23.6 \%$. Las mujeres fueron las más afectadas en ambos grupos en todos los niveles $(p=0.004)$. Los burócratas llegan al $23.85 \%$ de SA, los informales al $5.7 \%$, con diferencia significativa $(p=0.005)$. La tendencia sugiere que conforme se incrementa la edad disminuye la seguridad en ambos grupos. El mejor nivel socioeconómico (NSE) se asoció a mayor seguridad alimentaria con $\mathrm{Chi}^{2}$ de Pearson significativa $(p=.000)$. Prevaleció algún tipo de inseguridad alimentaria cuando los sujetos eran viudos o vivían solos con una asociación significativa de $p=0.006$. Conclusión: La inseguridad alimentaria es un problema significativamente mayor entre los trabajadores informales.
\end{abstract}

Palabras clave: Indicadores sociodemográficos, seguridad alimentaria, adultos mayores.

Food security and socio-demographic indicators in older adults. Objective. Identify and compare the association between sociodemographic indicators and food security (SA) in two groups of older adults: state workers and informal or self-employed workers. Method: Analytical and comparative crosssectional research in $>60$ years old, simple random sampling and stratified by age $(60-70,71-80$ and >81) inegrated by 134 and 123 subjects for the first and second group. An ad hoc questionnaire was applied for sociodemographic data, the AMAI Rule for the Socioeconomic Level and the Latin American and Caribbean Scale for Food Security (ELCSA). Results: female predominance in both institutions (56.7\% and $74.0 \%$; average age 68.5 and 67.8 years, respectively). In the Food Insecurity, there was a significant difference between both groups $(p=0.001$ ), in the bureaucrats there prevailed slight insecurity with $91 \%$ and moderate for the informal ones, $23.6 \%$. The women were the most affected in both groups at all levels $(p=0.004)$. Bureaucrats reach $23.85 \%$ of SA, informal workers reach $5.7 \%$, with significant difference $(p=0.005)$. The trend suggests that as age increases, security in both groups decreases. The best socioeconomic level (NSE) was associated with greater food safety with significant Pearson CHI2 $(P=.000)$. Some type of food insecurity prevailed when the subjects were widowers or lived alone with a significant association of $p=0.006$. Conclusion: Food insecurity is a significant problem among informal workers.

Key words: Demographic indicators, food security, older adults.

Correspondencia: Flor de María Sánchez Morales. Retorno Mina la Asturiana nº5. C.P.: 98610. Colonia Conde de Bernárdez. Guadalupe, Zacatecas (México). E-mail: florsan2270@gmail.com 
El proceso de envejecimiento de la población, una realidad en el mundo que genera cambios de consecuencias estructurales, económicas y sociales nunca antes vistas en la historia de la humanidad. Actualmente, el número de adultos mayores (AM) es dos veces superior al de 1980 y en 2050 habrá casi 395 millones de mayores de 80 años, cuatro veces más que ahora. La mayoría viven en países de ingresos bajos o medianos y en 2050, ese porcentaje ascenderá al 80\% (OMS, 2012). En México se estima que habrá 15 millones en 2020 equivalentes al $12.5 \%$ de la población, poco más de 22 millones en 2030 con el $17.5 \%$, y aumento a 36.5 millones o $28 \%$ en el 2050 (CONAPO, 2014). En Zacatecas los AM se han incrementado, llegando en 2014 al 10.2\%, para ocupar el cuarto lugar a nivel nacional (INEGI, 2014), y uno de los primeros con $65.6 \%$ de ellos en pobreza multidimensional (INEGI, 2015).

El envejecimiento puede ser visto como éxito social, ya que deriva de factores como el grado de urbanización, nivel de educación, desarrollo económico, progresos tecnológicos y médicos y las mejoras en el nivel de vida de la población; pero, trae aparejado varios retos a la sociedad, las familias y las instituciones, que implican mayor demanda de servicios y recursos no siempre posibles de satisfacer, sobre todo, para garantizarles una vida de calidad.

La vejez corresponde a un momento de la vida caracterizado por múltiples pérdidas: cambios en los ingresos económicos, pérdida de oportunidades de trabajo, de actividad e interacción social con su entorno, por lo que muchos resultan excluidos (Gerrig y Zimbardo, 2005), o en aislamiento (Moreno, Sánchez, y Doubova, 2017). Hay prevalencia importante de viudez, baja escolaridad, baja participación laboral y baja cobertura de pensiones con montos bajos.

Igualmente los cambios bio-psico-funcionales elevan su vulnerabilidad a enfermedades crónicas y afectan su salud, siendo los hábitos alimentarios un importante factor protector o de riesgo, según su calidad (Gómez, Vicente, Vila, Casajus, y Ara, 2012; García, Álvarez, y De Man, 2012), al igual que la actividad física como lo demuestra Parra Rizo (2017). Disminuyen las probabilidades de supervivencia y capacidad de reserva del individuo (Zea, López, y Valencia, 2008). Ello determina un grupo poblacional con mayores expectativas de vida, pero en condiciones físicas, mentales, sociales y nutricionales, no adecuadas (Encuesta de Calidad de Vida, 2006). Donde resalta la importancia de identificar y garantizar su seguridad alimentaria (SA).

El concepto de seguridad alimentaria, pactado en la Cumbre Mundial de la Alimentación 2006, en Roma, por la Organización de las Naciones Unidas para la Alimentación y la Agricultura (FAO, 2014) señala: "la seguridad alimentaria existe cuando todas las personas tienen, en todo momento, acceso físico, social y económico a alimentos suficientes, inocuos y nutritivos que satisfacen sus necesidades energéticas diarias y preferencias alimentarias para llevar a cabo una vida activa y saludable" (ONUAA, 2007), o bien "la situación en la que una población tiene en todo momento 
acceso físico y económico a suficientes alimentos para atender a sus necesidades nutricionales con el fin de llevar una vida productiva y sana" (FAO, 2002).

Entre los determinantes de la seguridad alimentaria están los factores sociodemográficos, considerados como el conjunto de variables económicas, laborales, sociológicas, y educativas, por las que se califica a un sujeto o colectivo dentro de una jerarquía social, y resultan determinantes de las condiciones de vida de los sujetos. En el perfil sociodemográfico se enlistan variables como el estatus socioeconómico, condiciones laborales, edad, sexo, estado civil, localidad de residencia (rural-urbano), y alfabetismo entre otras. Estas variables, además de influir de manera individual, generan distintos escenarios al incidir de manera conjunta (Millán-León, 2010).

Muchos AM mexicanos no cumplen con los requisitos de la SA, ya que el acceso a una alimentación adecuada en términos de cantidad, calidad e inocuidad es incierto o limitado, con efectos en el estado nutricional, calidad de vida y longevidad, que constituyen un problema de salud pública, ya que el estado físico, funcional y mental de los sujetos depende de la forma de alimentarse, desde la infancia hasta la edad adulta. Un AM se encuentra en situación de vulnerabilidad alimentaria cuando tiene factores que lo colocan en riesgo de inestabilidad alimentaria y nutricional, incluyendo sus habilidades de enfrentamiento (ONUAA, 2004). Por su parte Alwang (2001) comenta que es un estado de riesgo, motivado por condiciones económicas, sociales o ambientales, de que el acceso a suficiente alimento e inocuo, que satisfaga las necesidades nutricionales se vea reducido (Guardiola, 2006).

En México y Latinoamérica cada vez aumenta su vulnerabilidad pues las políticas relacionadas con la vejez, están permeadas por las crisis económicas en las que prevalece la pobreza, la desigualdad, y una baja cobertura y calidad de los sistemas de protección social. Son los ancianos quienes sufren más por circunstancias desfavorables de tipo económico, social, político, cultural y familiar, que se expresan en inseguridad alimentaria y un mal estado de salud (Wolfe, 2003).

De acuerdo con la Encuesta Nacional de Ocupación y Empleo 2014 (INEGI, 2014) la tasa de participación económica de la población general de AM en México fue de $33.7 \%$; en los hombres $50.7 \%$ y mujeres $19.4 \%$, la cual disminuye conforme avanza la edad, con pérdida de oportunidades laborales, lo que no viene compensado con una buena pensión o jubilación, esto los obliga a permanecer en el espacio laboral, ya sea posponiendo la jubilación o procurando ingresos complementarios (Ham, 2003), generalmente obtenidos en condiciones desfavorables, por su inserción en el trabajo informal, con ingresos abajo del salario mínimo.

Datos del Consejo Nacional de Evaluación de la Política de Desarrollo Social estimaron la proporción de hogares con niveles de inseguridad alimentaria moderada y severa con uno o más integrantes adultos mayores, llegando a $27.9 \%$. Sólo dos de cada diez pudieron solventar sus gastos (CONEVAL, 2010). Durante 2012, el 21.4\% de los AM 
presentaron carencia de acceso a la alimentación y no adquirieron lo indispensable para una nutrición adecuada. El 43.2\% de hombres y mujeres de 65 años o más se encontraban en situación de pobreza multidimensional, $10 \%$ en pobreza extrema, $72 \%$ vulnerables por carencias sociales, y $49.4 \%$ por ingreso (INEGI, 2013).

Los ancianos que viven en extrema pobreza, de baja escolaridad, indígenas o que nunca han trabajado, optan por consumir alimentos con bajo valor nutrimental o energético (caldos, atole de agua, verduras muy cocidas) por su precio accesible (Yuan et al., 2012). Zacatecas durante 2012 ocupaba el veinteavo lugar con proporción de inseguridad alimentaria moderada y severa de (28\%), al ser las categorías más graves, significa que algunos hogares tuvieron que disminuir la cantidad de alimentos consumidos o, peor aún, un adulto se quedó sin comer en todo un día (ENSANUT, 2012). A nivel nacional, $28.2 \%$ de hogares se clasificaron con IA moderada o severa, donde los hogares rurales, indígenas y de nivel económico bajo fueron los más afectados: 35.4, 42.2 y 49.5\%, respectivamente (ENSANUT, 2012).

La seguridad económica es un determinante social que contribuye a la seguridad alimentaria. Su ausencia conduce a consumos inferiores a $67 \%$ de la ingesta diaria recomendada, sobre todo, en AM que ingieren menos calorías, magnesio, calcio, cinc y vitaminas $\mathrm{E}, \mathrm{C}$ y $\mathrm{B}_{6}$. La pobreza como factor de riesgo de desnutrición es más probable que se origine en una población débil y vulnerable. La discapacidad funcional afecta el consumo dietético a cualquier edad (Rodríguez et al., 2009) y la calidad de vida como: la pérdida de la salud (Parra, 2017), el deterioro cognitivo (Navarro et al., 2014), la falta de fortalezas (Ramírez, Ortega y Martos, 2015) y los aspectos emocionales (Etxeberria, 2016), pero es más probable que ocurra en anciana/os que vivan solas y tengan menos recurso para compensar cualquier pérdida de función. La calidad de la dieta puede disminuir (Beate, 2010).

Las limitaciones económicas también generan problemas de seguridad alimentaria en términos de inocuidad. Hay personas que no tiran la comida, aunque esté en mal estado, porque es lo único que cuentan para alimentarse, escasos ingresos implican comida de peor calidad, los que además se ven recortados en caso de necesidad o enfermedad. La mala alimentación y el deterioro de ciertas funciones perceptivas incrementan los problemas alimentarios (Pelayo, 2008). Para que el anciano tenga buena salud es importante que satisfaga todas sus necesidades nutricionales. Aquellos que viven en hogares con inseguridad alimentaria tienden a percibir su salud física y mental como peor (Stuff et al., 2003).

Con IA anciano puede llegar a sufrir graves efectos en el estado nutricional, con pérdida de peso no intencional o involuntario y la desnutrición, que en esta etapa, contribuye a un declive progresivo de la salud, afecta el estado funcional y cognitivo, empeora la percepción de salud, así como el estado físico y mental (Stuff et al., 2004). Aumenta la mortalidad y disminuye la calidad de vida, sobre todo en ancianos enfermos 
y/o pobres, y las consecuencias psicológicas también se hacen evidentes como la depresión, disminución del interés en las interacciones sociales y cambios en el estado de ánimo (Velásquez, 2011).

En los trabajadores burócratas cubiertos por el Instituto de Seguridad y Servicios Sociales de los Trabajadores del Estado (ISSSTE) y los trabajadores autoempleados o informales cubiertos ahora por el Seguro Popular y atendidos en los Centros de Salud de los SSZ, tienen diferentes factores sociodemográficos, ya que estos últimos carecen de una pensión o jubilación que los respalde, por lo general son de escasos recursos y de baja escolaridad, mientras que los trabajadores del Estado reciben una jubilación o pensión, aunque no suficiente, pero segura. En ambos, se desconoce el grado de seguridad alimentaria que ostentan y sus diferencias.

Por ello el objetivo del presente trabajo fue identificar y comparar los indicadores sociodemográficos y seguridad alimentaria en adultos mayores de dos instituciones.

\section{MÉTODO}

Estudio transversal, analítico, comparativo en 257 adultos mayores, hombres y mujeres, 134 trabajadores del Estado y derechohabientes del ISSSTE y 123 trabajadores informales o autoempleados derechohabientes del Seguro Popular atendidos por los Servicios de Salud de Zacatecas; seleccionados mediante muestreo simple aleatorio y estratificado por edad (60-70, 71-80 y >81). Sus datos sociodemográficos se obtuvieron mediante cuestionario elaborado ad hoc, posterior a la prueba piloto y validación del instrumento.

Se aplicó la Regla AMAI (Asociación Mexicana de Agencias de Inteligencia de Mercado y Opinión 2011), para obtener el nivel de bienestar económico y social del hogar. Es un índice que clasifica a los hogares en siete niveles considerando cuatro dimensiones: Infraestructura práctica, Infraestructura sanitaria, Infraestructura básica, y Capital humano.

La Escala Latinoamericana y Caribeña para medir seguridad alimentaria (ELCSA), propuesta en 2007 como herramienta válida para registrar la experiencia de IA dentro de los hogares de ámbito regional (Álvarez, Estrada, Montoya, y Quiñonez, 2008; Carmona, Paredes, y Pérez, 2017). Uno de los métodos más utilizados por encuestas nacionales y el único que se basa en la experiencia o percepción de los individuos y los hogares (Pérez-Escamilla y Segall-Correa, 2008). Consta de 15 preguntas dicotómicas dirigidas al jefe o jefa de familia o a la persona encargada de preparar los alimentos en el hogar, con periodo de referencia de tres meses, clasifica los resultados en: seguridad alimentaria, e inseguridad alimentaria leve, moderada y severa. 
Previa obtención de la autorización de las autoridades y del consentimiento informado de lo sujetos seleccionados se aplicaron las entrevistas por el investigador en las áreas de consulta de las instituciones de salud. Las comisiones de ética e investigación de ambas instituciones revisaron y aprobaron el protocolo. La información obtenida fue capturada y analizada mediante SPPS 21.0. Se realizó análisis descriptivo y de asociación por $c h i^{2}$ de Pearson, y para el estudio de las diferencias de medias se realizó un contraste no paramétrico de $U$ de Mann-Whitney para comparar las instituciones.

\section{RESULTADOS}

Los resultados corresponden a los 257 AM, 65\% (167) mujeres y 35\% (90) hombres. Del total, 134 fueron trabajadores del estado y 123 trabajadores autoempleados o informales. El nivel socioeconómico predominante fue el medio y alto para los trabajadores del Estado y el bajo para los trabajadores informales. En la distribución según sexo, existió una alta prevalencia de mujeres en ambos grupos, en autoempleo también dominaron las mujeres $(74 \%)$ y varones $(26 \%)$, en cambio, entre burócratas, fue más equilibrada $56.7 \%$ mujeres y $43.3 \%$ hombres.

El promedio de edad en los trabajadores del estado fue de 68.5 años con una desviación estándar $(D E)$ de \pm 6.9 años y un rango de 60 a 90 años, y en los informales una media de 67.8 años $D E \pm 7.6$ y un rango de 60 a 92 años y en su mayoría casados o viudos.

El análisis de la seguridad alimentaria en relación al tipo de inserción laboral indicó que los trabajadores del Estado tienen seguridad alimentaria en casi la mitad de la muestra, en comparación con los informales donde sólo se alcanzó el 12.2\% (15). El grado de inseguridad leve predominó en los informales con poco más de la mitad 51.2\% (63), contra $41 \%$ (55) de los burócratas.

La inseguridad moderada disminuye para ambos pero sigue siendo mayor en los autoempleados con casi el doble de adultos mayores (33 contra 17) de los burócratas, éstos no presentaron ningún caso de inseguridad severa, mientras que los trabajadores informales reportaron un $9.8 \%$ (12). La prueba no paramétrica para comparar dos muestras $U$-Mann Whitney, indicó que en promedio existió asociación significativa $(p=0.000)$ entre el grado de seguridad alimentaria y la pertenencia a las instituciones, con mejores condiciones alimentarias en los trabajadores del Estado (Figura 1). 
" Trabajadores del Estado $₫$ Trabajadores Autoempleados e informales

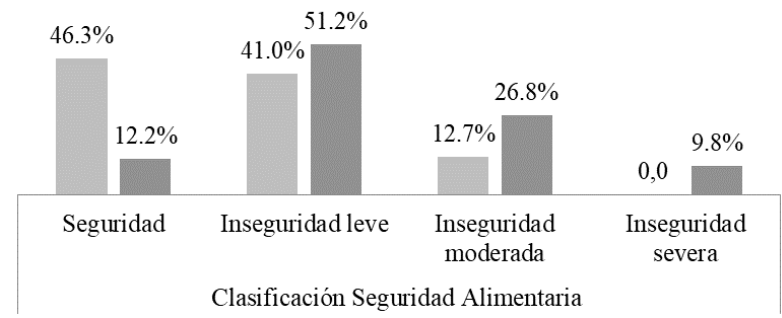

Figura 1. Seguridad alimentaria por inserción laboral de los adultos mayores

En el nivel socioeconómico alto se presentó el mayor porcentaje de seguridad alimentaria de la muestra, un porcentaje muy bajo de inseguridad leve y aun menor de moderada, sin presentar casos de inseguridad severa. En el nivel socioeconómico medio la seguridad alimentaria baja de 28.4 a $17.9 \%$, mientras que la inseguridad leve predomina con el más alto porcentaje de la muestra y la moderada disminuye de 0.7 a $6.0 \%$. Por el contrario, en el nivel socioeconómico bajo no existe seguridad alimentaria, solamente están representadas la inseguridad moderada y leve. La prueba de contraste $C h i^{2}$ de Pearson llevó a la conclusión de que el grado de nivel socioeconómico influye en la seguridad alimentaria $(p=0.000)$ (Figura 2).

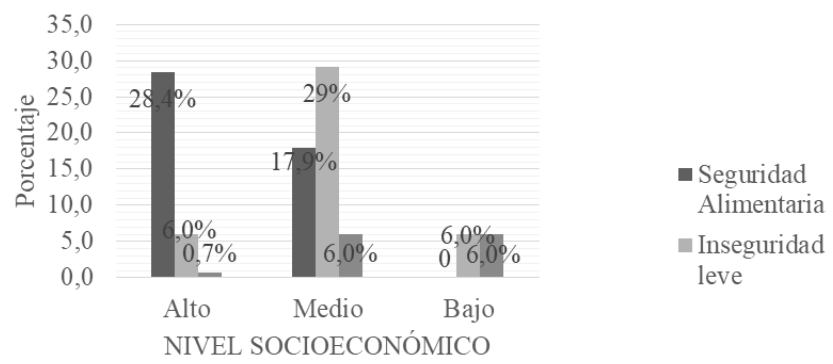

Figura 2. Nivel socioeconómico y grado de seguridad alimentaria en trabajadores del Estado

En trabajadores informales se encontró que tan solo el 1.6\% se ubicó en el nivel socioeconómico alto, sin seguridad alimentaria en ninguno de ellos, solamente inseguridad leve; en el nivel medio, menos del $10 \%$ tuvieron seguridad alimentaria, casi una quinta parte presentó inseguridad leve y aunque en bajo porcentaje se presentaron casos de inseguridad moderada y severa; en el nivel bajo disminuye de manera importante la seguridad alimentaria hasta un $2.4 \%$ y se incrementan la leve, moderada y severa. En este grupo aparece el nivel socioeconómico marginal, donde desaparece la seguridad alimentaria y disminuyen la inseguridad alimentaria leve, moderada y severa (Figura 3). La prueba de contraste $C h i^{2}$ de Pearson llevó a la conclusión de que el grado 
de nivel socioeconómico influye en la seguridad alimentaria, existió una asociación entre las variables $(p=0.000)$.

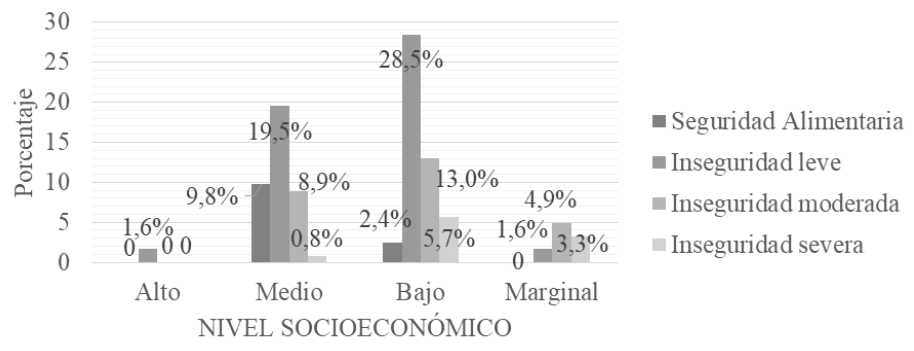

Figura 3. Nivel socioeconómico y grado de seguridad alimentaria en trabajadores informales

En influencia del sexo para la seguridad alimentaria se encontró que las mujeres sufren más de inseguridad que los varones y lo hacen en todos los niveles y en ambos grupos. Entre los sujetos trabajadores del estado predomina la seguridad, con una distribución más o menos homogénea para hombres y mujeres, aun cuando a medida que se incrementa el grado de inseguridad disminuye la presencia masculina. Mientras que entre los trabajadores informales tanto en hombres y mujeres el porcentaje disminuye conforme se agrava la inseguridad alimentaria, pero las mujeres están presentes con porcentajes más altos que los varones (Figura 4). En promedio el grado de seguridad alimentaria fue diferente entre ambos con evidencia significativa $(p=0.004)$. Siendo peor las condiciones de la mujer.

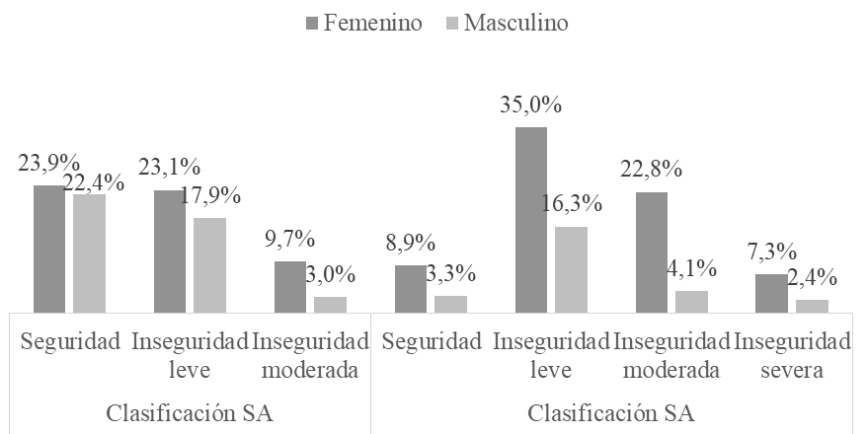

Figura 4. Grado de Seguridad Alimentaria por sexo e inserción laboral

En el análisis por grupos de edad de los trabajadores del estado, fue en el grupo más joven de 60-70 años donde se concentró el mayor porcentaje de seguridad alimentaria, el cual disminuye drásticamente en el grupo de 71-80 años, y aún más en los mayores de ochenta donde sólo llega al $1.5 \%$, este comportamiento se repite en los otros grupos con 
diferentes grados de inseguridad alimentaria, sin haber inseguridad severa. Para los AM del sector informal el grupo de 60-70 presenta una importante disminución de seguridad alimentaria en comparación a los trabajadores del ISSSTE, en ellos se concentran los porcentajes más altos de seguridad leve y en todos los grupos de edad aparece la inseguridad severa (Figura 5). Se encontró evidencia significativa entre la asociación de algún grado de seguridad alimentaria y la edad $(p=0.005)$.

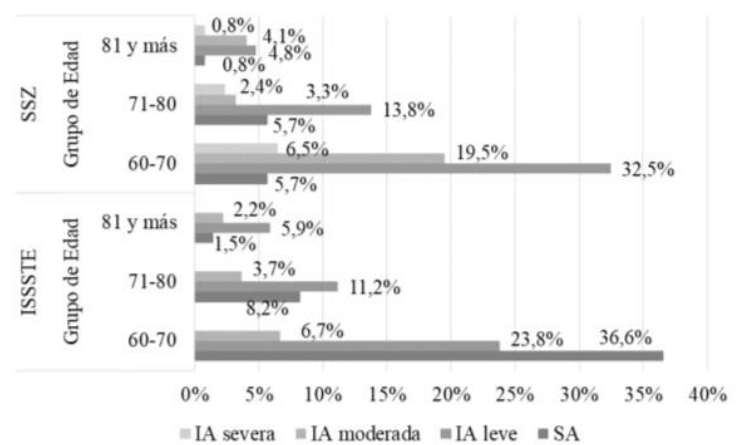

Figura 5. Seguridad alimentaria por grupos de edad e inserción laboral

La seguridad alimentaria fue mayor en los AM casados, cuyo porcentaje disminuyó con el incremento de la severidad tanto en la moderada como la leve.

En los autoempleados el porcentaje de seguridad es muy baja pero los casados tienen el más alto porcentaje, al igual que en la inseguridad leve, moderada y severa, seguidos por los viudos y en poca cantidad los solteros. Prevaleció algún tipo de inseguridad alimentaria cuando éstos eran viudos o vivían solos con una asociación significativa de $p=0.006$.

Entre los trabajadores del estado se observó el mayor porcentaje de seguridad alimentaria con (35.1\%) casados, mientras que en los informales el mayor porcentaje fue para la inseguridad leve (33.3\%) en los casados también (Figura 6). 


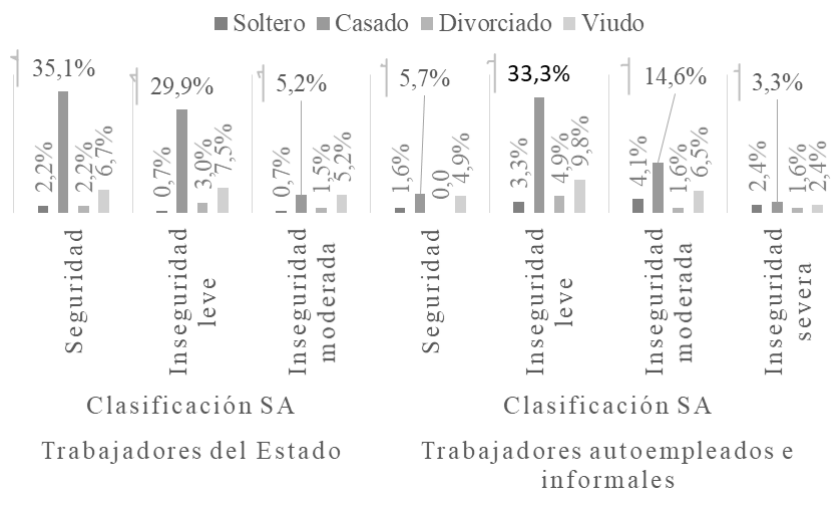

Figura 6. Seguridad alimentaria por estado civil e inserción laboral

\section{DISCUSIÓN}

La prevalencia de seguridad alimentaria en la muestra fue de $33.9 \%$ y los que tuvieron algún grado de inseguridad llegó al $66.1 \%$ superior al encontrado en adultos mayores chilenos de 40.4\% (Vargas, Alvarado, y Atalah, 2013), lo que resulta interesante dados los altos índices de sobrepeso y obesidad en este grupo poblacional, lo que orienta a pensar que el problema alimentario de los AM zacatecanos es más de calidad que de cantidad (Restrepo, Morales, Ramírez, López, y Varela, 2006). Lo anterior está en relación con el grado de inseguridad leve que predominó y que implica un bajo acceso a los alimentos de calidad. Con un alto consumo de ingesta de alimentos de bajo costo, alta densidad energética y ricos en carbohidratos debido a su bajo costo (Mendoza et al., 2017).

La inseguridad alimentaria mostró diferencias significativas entre los burocratas y los autoempleados, con mejores condiciones para los primeros, lo que puede exlicarse por la diferencias socioecónomicas entre ambos, los del ISSSTE cuentan con salario y prestaciones sociales, si aun trabajan, o bien, tienen asegurada una jubilación. Mientras que los autoempleados carecen de ambas y si trabajan (que son los más), lo hacen en el sector informal con salarios bajos, e inseguros.

Aunque la SA plantea problemas de producción y distribución, es sobre todo, un problema de ingresos que permitan el acceso a una alimentación decente, lo que está unido con el trabajo (Bonnal, Losch, Marzin, y Parrot, 2013). Como señala Wolfgan el mayor riesgo para desarrollar inseguridad alimentaria esta motivado por condiciones económicas adversas o bajos ingresos familiares (Vargas Puello et al., Alvarado y Atalah, 2013), lo que es evidente en estos resultados. 
Se observó diferencia significativa de la seguridad alimentaria entre los dos grupos con inserción laboral diferente, con beneficio de los burócratas. A mayor nivel socioeconómico mayor seguridad alimentaria, a menor nivel socioeconómico se incrementa el grado de inseguridad. Los resultados son también coincidentes con la literatura en que son las mujeres las más sufren esta situación y de que la seguridad alimentaria disminuye con los años, sobre todo en las personas enfermas y solas.

\section{CONCLUSIONES}

La inseguridad alimentaria es un mayor problema entre los trabajadores informales o autoempleados que en los trabajadores del Estado. Dado que la población de estudio no está considerada como de alta pobreza, la prevalencia de inseguridad es alta, y más bien relacionada con el consumo de productos alimenticios de mala calidad por su bajo costo, sobre todo, en las mujeres y personas solas que deben ser de atención especial. La fuerte asociación con factores socio-demográficos, económicos y nutricionales, lo pone como tema relevante en las políticas públicas, sociales, de salud y de investigación.

\section{REFERENCIAS}

Consejo Nacional de Evaluación de la Política de Desarrollo Social, CONEVAL, (2010). Día del adulto mayor. México, D.F. Recuperado en http://www.coneval.gob.mx/rw/ resource/coneval/diadeladultomayor.pdf. Consultado abril 2013.

Consejo Nacional de Población y Vivienda, CONAPO. (2014). Proyecciones de población 2010-2050. Secretaria de gobernación, México. Recuperado en http://www.conapo.gob.mx/es /CONAPO /Proyecciones.

Departamento de Epidemiología del Ministerio de Salud. Gobierno de Chile (2006). Encuesta de Calidad de Vida. Disponible en http://epi.minsal.cl/wpcontent/uploads /2012/07/II-EncuestadeCalidad-de-Vida-y-Salud-2006.pdf. Consultado diciembre 2016.

Instituto Nacional de Salud Pública, INSP. (2012). Encuesta Nacional de Salud y Nutrición 2012, ENSANUT 2012. Estado de nutrición, anemia, seguridad alimentaria en la población mexicana, México. Recuperado en http://ensanut.insp.mx/doctos/ENSANUT2012 _Nutricion.pdf. Consultado mayo 2015.

INSP. Encuesta Nacional de Salud y Nutrición. (2012), ENSANUT 2012. Apartado Zacatecas. Estado de nutrición, anemia, seguridad alimentaria en la población zacatecana México. Recuperado en http://ensanut.insp.mx/doctos/ENSANUT2012_Nutricion.pdf. Consultado mayo 2015.

INEGI. (2013). Estadísticas a propósito del día mundial de la justicia social, Aguascalientes, Ags. Recuperado en http://www.inegi.org.mx/inegi/contenidos/espanol/prensa/Contenidos /estadisticas.justicia.pdf. Consultado mayo 2015.

INEGI. (2014). Encuesta Nacional de Ocupación y Empleo 2014, ENOE. Recuperado en: www.inegi.org.mx $>$ Estadísticas $>$ Encuestas en hogares.

Instituto Nacional de Estadística y Geografía, INEGI. (2014). Mujeres y hombres en México, pp. 20-21. Recuperado en http://cedoc.inmujeres.gob.mx/ documentos download/101239.pdf

Instituto Nacional de Estadística y Geografía, INEGI (2015). Cuéntame, Información por Entidad, México. Recuperado en http://www.cuentame.inegi.org.mx/monografias/ informacion/zac/poblacion/dinamica.aspx?tema $=$ me $\&$ e $=32$ 
Organización de las Naciones Unidas para la Alimentación y la Agricultura (FAO) (2002). The estate of Food insecurity in the world. Recuperado en: http://ftp.fao.org/docrep /fao/005/y7352e/y7352e00.pdf

Organización de las Naciones Unidas para la Agricultura y la Alimentación. FAO (2004). El estado de la inseguridad alimentaria en el mundo. Roma: FAO. Recuperado en www.fao.org/docrep/fao/007/y5650s /y5650s00.pdf.

Organización de las Naciones Unidas para la Alimentación y la Agricultura, ONUAA (2007). Conferencia Internacional sobre la agricultura orgánica y seguridad alimentaria, Roma. Recuperado en http://www.fao.org/organicag/oa-specialfeatures/oa-foodsecurity/es/

Organización Mundial de la Salud, OMS (2012). Día Mundial de la Salud 2012-¿Está usted preparado? Recuperado en http://www.who.int/world-health-day/2012/toolkit/background/es /

Organización de las Naciones Unidas para la Alimentación y la Agricultura (FAO) (2014). La Declaración de Roma sobre la Nutrición. Recuperado en: http://www.fao.org /resources/infographics/infographics-details/es/c/266120/

Álvarez, M.C., Estrada, A., Montoya, E.C., y Melgar-Quiñónez, H. (2006). Validación de escala de la seguridad alimentaria doméstica en Antioquia, Colombia. Salud Pública Mexicana, 48, 474481.

Alwang, J., Siegel, P.B., y Jorgensen, S.L. (2001). Vulnerability: a view from different disciplines. Social protection discussion paper series, 115. The World Bank. Washington DC.

Arango, D.C., y Peláez, E. (2012). Envejecimiento poblacional en el siglo XXI: oportunidades, retos y preocupaciones. Salud Uninorte. 28(2), 335-348. Recuperado en: http://www.scielosp.org/scielo.php?script=sci_nlinks\&pid=S08643466201700010001600002 $\& \operatorname{lng}=$ en

Beate, U. (2010). Nutrición y adultos mayores, In: J. Brown. Nutrición en las diferentes etapas de la vida, Tercera edición. (pp.467-469). Mc Graw Hill, México.

Bonnal, P., Losch, B., Marzin, J., y Parrote, L. (2013) Desafios de la pobreza, del empleo y la seguridad alimentaria. Las agriculturas familiares y los mundos del futuro. Ed. J.M. Rourisseau, (pp. 187206) Agence Francaise for developpement. Recuperado en http://agritrop.cirad.fr/583929/7/583929.pdf

Carmona, S.J., Paredes, S.J., y Pérez, S.A. (2017). La Escala Latinoamericana y del Caribe sobre Seguridad Alimentaria (ELCSA): Una herramienta confiable para medir la carencia por acceso a la alimentación. Revista Iberoamericana de las Ciencias Sociales y Humanísticas, RICSH, 6(11), 1-24.

Etxeberria, I. (2016). Estudio descriptivo de algunas variables emocionales en las personas mayores. European Journal of Investigation in Health, Psychology and Education, 6(2), 57-69.

García, A., Álvarez, J., y De Man, F. (2012). Envejecimiento y desnutrición; un reto para la sostenibilidad del SNS; conclusiones del IX Foro de Debate Abbott-SENPE. Nutrición Hospitalaria, 27(4), 1060-1064.

Gerrig, J.R., y Zimbardo, G.P. (2005). Psicología y vida. Editorial books.

Gómez, A., Vicente, G., Vila, S., Casajus, J., y Ara, I. (2012). Envejecimiento y composición corporal: la obesidad sarcopénica en España. Nutrición Hospitalaria, 27(1), 22-30.

Guardiola, J. (2006). La seguridad alimentaria: estimación de índices de vulnerabilidad en Guatemala, en VIII Reunión de Economía Mundial, Provincia de Alicante. Recuperado en http://altea.daea.ua.es/ochorem/comunicaciones/MESA2COM/GuardiolaGonzálezVivero2.pd f.

Ham, R., (2003). Envejecimiento en México: el siguiente reto de la transición demográfica. El Colegio de la Frontera Norte, Tijuana.

Lo, Y.T., Chang, Y.H., Lee, M.S., y Wahlqvist, M.L. (2012). Dietary diversity and food expenditure indicators of food security in older Taiwanese. Appetite, Taiwan, 58, 180-187.

Millán-León, B. (2010). Factores asociados a la participación laboral de los adultos mayores mexiquenses. Papeles de población, 16(64), 93-121. Recuperado en: http://www.scielo. 
org.mx/scielo.php?script=sci_arttext\&pid=S1405-52010000200005\&lng=es\&tlng=es. Consultado marzo 2017.

Mendoza, A., Pérez, A.E., Aggarwal, A., y Drewnowski, A.(2017). Energy density of foods and diets in Mexico and their monetary cost by socioeconomic strata: analyses of ENSANUT data 2012. Journal Epidemiology Community Health, 71,713-721.

Moreno, T.K., Sánchez, G.S., y Doubova, S.V. (2017). Factores asociados con el aislamiento social en una muestra de adultos mayores con seguridad social. Salud pública Mexicana, 59(2), 119-120. Recuperado en: http://dx.doi.org/10.21149/8078.

Navarro, E., Calero, M.D., y Calero García, M.J. (2014). Diferencias entre hombres y mujeres mayores en funcionamiento cognitivo y calidad de vida. European Journal of Investigation in Health, Psychology and Education, 4(3), 267-277.

Parra-Rizo, M., (2017). Componentes de influencia más valorados en la calidad de vida por las personas mayores de 60 años físicamente activas. European Journal of Investigation in Health, Psychology and Education, 7(3), 135-144.

Pelayo, M. (2008). La seguridad alimentaria en la tercera edad. España, 2008. Recuperado en http://www.consumer.es/seguridad-alimentaria/. Consultado marzo 2014.

Pérez-Escamilla, R., Correa-Segall, A., Kurdian, L., Archanjo, M., Marín-León, L. y Panigassi, G. (2004). An Adapted Version of the U.S. Department of Agriculture Food Insecurity Module Is a Valid Tool for Assessing Household Food Insecurity in Campinas, Brazil, The Journal of Nutrition, 134, 1923-1928.

Ramírez, E., Ortega, A., y Martos R., (2015). Las fortalezas en personas mayores como factor que aumenta el bienestar. European Journal of Investigation in Health, Psychology and Education, 5(2), 187-195.

Restrepo, S., Morales, R., Ramírez, M., López, M., y Varela, L. (2006). Los hábitos alimentarios en el adulto mayor y su relación con los procesos protectores y deteriorantes en salud. Revista chilena de Nutrición, 33(3), 500-510.

Rodríguez, Q.T., Fabelo, R., Justo, R., e Iglesias, M. (2017). Barreras percibidas en los servicios comunitarios y de salud por los adultos mayores. Revista Cubana de Salud Pública, 43(1), 1626. Recuperado en: http://www.scielosp.org/scielo.php?script=sciarttext\&pid $=$ S08644662017000100016\&lng=en\&tlng=pt. Consultado Septiembre 2017.

Stuff, J., Casey, P., Szeto, K., Gosett, J., Robbins, J., Simpson, P., Connell, C., y Bogle, M. (2004). Household Food Insecurity Is Associated with Adult Health Status. Journal Nutricional, 34(9), 2330-2335. Recuperado en: http://jn.nutrition.org/content/134/9/2330.full. Consultado septiembre 2017.

Vargas, P.V., Alvarado, O.S., y Atalah, E. (2013). Inseguridad alimentaria en adultos mayores en 15 comunas del Gran Santiago: un tema pendiente. Nutrición Hospitalaria, 28(5), 1430-1437. Recuperado en: https://dx.doi.org/10.3305/nh.2013.28.5.6663

Velásquez, M.C. (2011). Desnutrición en los adultos mayores: La importancia de su evaluación y apoyo nutricional. Revista de Salud Pública y Nutrición, 12(2), 1. Recuperado en http://www.respyn.uanl.mx/xii/2/ensayo/ensayo_des_adult_may.htm

Wolfe, W., Frongillo, E. y Valois, P. (2003). Understanding the experience of food insecurity by elders suggests ways to improve its measurement. The Journal of Nutrition, United States, 133, 27622769.

Zea, M.C., López, M.E., Valencia, C.M., Soto, J.A., Camilo, D., Lopera, F., y Roland, W.D. (2008). Autoevaluación de calidad de vida y envejecimiento en adultos con riesgo de Alzheimer. Red de Revistas Científicas de América Latina Redalyc, 26(1), 24-35. Medellín Colombia. Recuperado en http://www.scielo.org.co pdf/iee/v26n1/v26n1a02.pdfn.

Recibido: 27 de noviembre de 2017 Recepción Modificaciones: 14 de diciembre de 2017 Aceptado: 20 de enero de 2018 